\title{
The Relationship Between the Dietary Inflammatory Index and Metabolic Syndrome in Ravansar Cohort Study
}

This article was published in the following Dove Press journal:

Diabetes, Metabolic Syndrome and Obesity: Targets and Therapy

\author{
Hadi Abdollahzad (D) \\ Yahya Pasdar (D) ${ }^{\prime}$ \\ Seyed Mostafa Nachvak' \\ Shahab Rezaeian (D) ${ }^{2}$ \\ Amir Saber (D) ${ }^{\prime}$ \\ Razieh Nazari ${ }^{1,3}$ \\ 'School of Nutrition Sciences and Food \\ Technology, Kermanshah University of \\ Medical Sciences, Kermanshah, Iran; \\ ${ }^{2}$ Department of Epidemiology, School of \\ Health, Kermanshah University of \\ Medical Sciences, Kermanshah, Iran; \\ ${ }^{3}$ Student Research Committee, \\ Kermanshah University of Medical \\ Sciences, Kermanshah, Iran
}

Purpose: The aim of this study was to investigate the association between dietary inflammatory index (DII) and metabolic syndrome (MetS) and its components using data of Ravansar non-communicable diseases (RaNCD) cohort study.

Patients and Methods: The present cross-sectional study was performed using the information of 6538 participants in the RaNCD study in Iran. A validated 125-item food frequency questionnaire (FFQ) was used to acquire DII scores. MetS was defined based on national cholesterol education program-adult treatment panel III (NCEP-ATP III) criteria. The association between DII and MetS and its components was investigated by the logistic regression model using STATA software.

Results: A significant association was found between DII and MetS (OR trend: $1.08,95 \%$ CI: $1.01-1.15, \mathrm{P}=0.017)$, triglyceride $(\mathrm{TG})(\mathrm{OR}$ trend $1.06,95 \% \mathrm{CI}: 1.00-1.12, \mathrm{P}=0.030)$, fasting blood glucose (FBG) (OR trend: 1.10, 95\% CI: 1.01-1.20, $\mathrm{P}=0.018$ ) and high density lipoprotein cholesterol (HDL-C) (OR trend: 1.07, 95\% CI: 1.02-1.12, P=0.005) after adjustment for all covariates. Also, there was a significant relationship between DII score and waist circumference (WC) (OR trend: 1.07, 95\% CI: 1.01-1.14, $\mathrm{P}=0.016$ ).

Conclusion: Higher DII score (a pro-inflammatory diet) had a significant association with the risk of MetS and its components, even after adjustment for different potential confounding factors including socio-demographic data and lifestyle habits. However, further longitudinal investigations with more dietary parameters are needed to elucidate the role of the pro-inflammatory diet in the etiology of MetS.

Keywords: dietary inflammatory index, metabolic syndrome, food frequency questionnaire, pro-inflammatory diet

\section{Introduction}

MetS is a set of risk factors that predisposes individuals to type 2 diabetes and cardiovascular disease (CVD), also it imposes many economic burdens on the health system. ${ }^{1,2}$ These risk factors consist of hypertension, insulin resistance, dyslipidemia, and abdominal obesity. People with MetS are 2-4 times more likely to be exposed to stroke, 3-4 times the increased risk of myocardial infarction and death regardless of previous history of CVD compared to those without MetS. The prevalence of MetS (based on NCEP-ATPIII, 2001) varies from $8 \%$ to $43 \%$ in men and $7 \%$ to $56 \%$ in women worldwide. ${ }^{3}$ According to an epidemiological data from Middle Eastern, the prevalence of MetS in men was 20.7-37.2\% and in women $32.1 \%$ to $42.7 \%$. ${ }^{4}$ As well, according to NCEP-ATPIII criteria, the overall
Nazari

Department of Nutrition, Faculty of Nutritional Sciences and Food

Technologies, Isar Sq., Across from Farabi Hospital, P.O. Box 67/985I35I,

Kermanshah, Iran

Tel +98-83 37/02009

Fax +98-83 37102002

Email amir.saber@kums.ac.ir;

roz.sahreh@gmail.com 
prevalence of MetS in Iran was $29 \%$, which in men and women was $24 \%$ and $35 \%$, respectively. ${ }^{5}$ Indeed, it seems that more than one-third of the Iranian adult population are exposed or have MetS problem. ${ }^{6}$

MetS is associated with a pro-inflammatory situation along with the increment of plasma concentrations of several inflammatory biomarkers including high-sensitivity C-reactive protein (hs-CRP), interleukin-6 (IL-6), and Tumor Necrosis Factor- $\alpha($ TNF- $\alpha){ }^{7}$ The inflammation is the response of the body to tissue damage or the presence of inflammatory stimuli. ${ }^{8}$ Numerous studies have shown that chronic exposure to a source of chronic inflammation gradually makes a mild inflammatory response and increases the risk of obesity, insulin resistance, diabetes, MetS, cardiovascular disease, or cancer. ${ }^{9}$ A healthy diet with a lower pro-inflammatory score may play a protective role against developing chronic diseases like MetS and diabetes. ${ }^{10}$ To evaluation of the inflammatory potential of a diet on human health, the DII was designed by researchers at the University of South Carolina. The DII is the first dietary index which used in different studies and assesses the pro-inflammatory and anti-inflammatory properties of the diet in any population by any type of dietary assessment method. ${ }^{11}$ The effects of different dietary patterns on inflammatory status assess through some pro-inflammatory biomarkers such as; TNF- $\alpha, \mathrm{CRP}$ and cell adhesion molecules (CAM) ${ }^{12-14}$ Besides, the validity of the DII was evaluated by two different food assessment methods ( $24 \mathrm{hrs}$ food recall and 7-day recall questionnaire) and showed that DII can predict the hs-CRP concentrations with the amount of $\leq 3 \mathrm{mg} / \mathrm{L}$. As well, the high DII score (pro-inflammatory diet) was associated with hs-CRP above $3 \mathrm{mg} / \mathrm{L} .{ }^{15}$

The DII categorizes the individual's diet in a range of maximally anti-inflammatory to maximally proinflammatory. ${ }^{11}$ Indeed, the higher DII score which is accompanying with an unhealthy diet represented the higher inflammatory effects of the diet and vice versa. ${ }^{16}$ The favorable effects of a healthy diet which reflects in anti-inflammatory properties are related to some foods and components like; fruits, vegetables, olive oil, red wine, nuts and polyphenols. ${ }^{17,18}$ On the other hand, the adverse effects of an unhealthy diet are attributed to high-fat dairy, butter, red meat, processed foods and saturated and trans fatty acids. ${ }^{19,20}$ Accordingly, numerous investigations with inconsistent results have been performed about the association of the DII and MetS in various populations. However, due to the inconsistent results of previous studies, it seems that further studies in diverse cultures and populations are needed to elucidate the association between DII score and MetS and its components. To our knowledge, it is the first study in Iran that evaluated the association of DII with MetS and its components using large data that collected by the RaNCD cohort study. Therefore, the aim of this study was to assess the association between DII and the prevalence of MetS using large data from the Iranian population.

\section{Materials and Methods Study Design and Subjects}

This cross-sectional study was performed using RaNCD cohort study data. The RaNCD study is a population-based prospective study that has been conducted on the 10,065 permanent residents consisted of men and women aged 35-65 years to investigate the results of chronic diseases and death in Ravansar (one of the cities of Kermanshah province in Iran) since $2014 .{ }^{21}$ This study is one of the parts of Iran's mega-cohort study PERSIAN (Prospective Epidemiological Research Studies in Iran). ${ }^{22}$ Ravansar, a region with urban and rural areas is located in western Iran in the Kermanshah province near the Iraq border which has about 50,000 populations with Kurdish ethnicity. The PERSIAN cohort study has been set up in 10 geographical regions in Iran. These areas have been selected based on local disease patterns, risk factors, population stability and local mortality and morbidity. The main focus of clinical researches is on prevention of chronic diseases, which comprises about $75 \%$ of the diseases and causes of mortality in the country.

All of the participants of RaNCD cohort study were permanent residents of this area. The inclusion criteria for participant recruitment were as follows: 1) age 35-65 years; 2) minimum residence of 1 year in Ravansar, with a minimum stay of 9 months per year; 3) likelihood of staying in Ravansar for the foreseeable future, together with willingness to participate; 4) provision of written informed consent; 5) capability to communicate with the research team and having Iranian citizenship. After performing a pilot study, the main phase of the study started in March 2015. From 15,000 people aged 35-65 years who live in both urban and rural areas of Ravansar district, the investigators decided to include 10,000 people based on all available resources and in agreement with the central PERSIAN team. To increase the feasibility of the study, people were recruited from both urban and rural areas. The size of the sample recruited to the study was proportional to the total population covered by each health center. The protocol details of this study were presented in previous 
studies. ${ }^{21}$ In this study, 10,065 individuals (4775 men and 5290 women) with available baseline MetS data were evaluated. MetS was defined using the NCEP-ATPIII criteria. ${ }^{23}$

Individuals with uncompleted baseline data $(\mathrm{n}=284)$, with cardiovascular disease $(\mathrm{n}=904)$, cancer $(\mathrm{n}=75)$, liver disease $(\mathrm{n}=424)$, thyroid disease $(\mathrm{n}=612)$, inflammatory disease $(\mathrm{n}=799)$, alcohol drinkers $(\mathrm{n}=232)$, and people who were addicted and drug abusers $(n=197)$ were excluded from the study. Finally, 6538 subjects (2931 men and 3607 women) were included in the study. The complete flowchart of inclusion and exclusion criteria has been presented in Figure 1.

\section{Data Collection}

In order to perform this study, socio-demographic characteristics, anthropometric indices, biochemical parameters, blood pressure, and nutritional status of all participants were collected from the RaNCD cohort study. All information was collected by trained health professionals at the study site, Ravensar (one of the cities of Kermanshah province in Iran), as following.

\section{Demographic Data}

All of the demographic data including age, gender, educational level, economic status, marital status, place of residence, physical activity; smoking, specific disease history (heart attack, stroke, various types of cancers), and common drug use were collected. Based on the marital and economic status (wealth), participants were divided into 3 and 5 groups: single, married, divorced and excellent to poor, respectively. The socioeconomic status of participants was evaluated by a comprehensive questionnaire about employment status, education, employment history, marital status, number and type of marriages (first-degree or seconddegree familial marriage or none), spouse's job, region of residency, number of domestic and international trips, access to landline and mobile phones, the internet and the extent of cell phone use. Also, participants according to their educational status, the number of study years, were categorized into 5 groups (illiterate, 1-5, 6-9, 10-12 and above 13) and based on tobacco use were divided into two groups as yes and no.

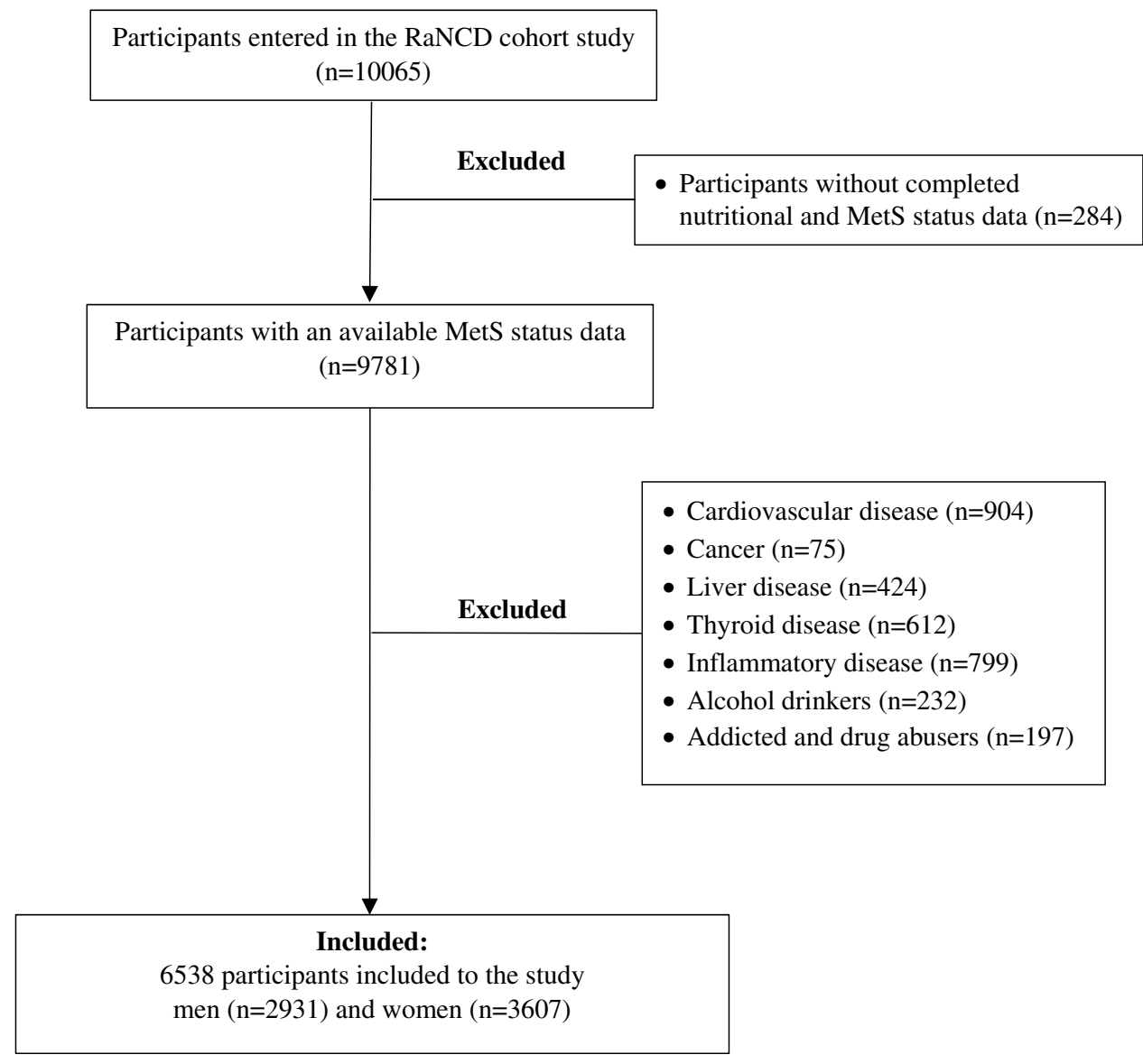

Figure I Flowchart with inclusion and exclusion criteria of participants. 
To evaluation of the physical activity status, the short form of International Physical Activity Questionnaire (IPAQ) has been used. According to this evaluation index, participants were divided into three groups using daily METs and determined cutoff points with low (24-36.5), moderate (36.6-44.9) and severe $(\geq 45)$ activity levels. ${ }^{21}$

\section{Anthropometric Indices, Biochemical Parameters and Blood Pressure}

For body mass index (BMI) calculation, the weight in light clothes and height was measured according to standard protocols with InBody 770 device (Inbody Co, Seoul, Korea) and automatic height gauge BSM 370 (Biospace Co., Seoul, Korea) respectively. BMI was calculated through dividing the weight $(\mathrm{kg})$ to height $\mathrm{t}^{2}\left(\mathrm{~m}^{2}\right)$.

The waist circumference was measured with light clothing at a level midway between the lowest rib cage and the iliac crest in expiratory condition. Systolic and diastolic blood pressure were measured using standard manual sphygmomanometer (Riester), after at least 4-5 mins of rest in sitting position, two times from both arms and the interval between two measurements was 10 mins. The mean of the two obtained blood pressure from both arms (left and right) were recorded as the final blood pressure. Blood samples of all subjects were collected after 12-hr fasting. After serum isolation by centrifuging at $2000 \mathrm{rpm}$ for $10 \mathrm{mins}$ at room temperature, blood samples and isolated serums were kept at $-80^{\circ} \mathrm{C}$.

\section{Diet and Nutritional Data}

Nutritional status was assessed using the validated national Iranian Food Frequency Questionnaire (FFQ). The FFQ assessment asks about consumption of food and drink during the past year. The questionnaire includes 125 food items appropriate for the Iranian population, including bread, cereal, grains, meat and meat products, milk and dairy products, vegetables, fruits, types of oil and oilseeds, sugar, miscellaneous food products, spices and food supplements. Also, some local foods were added into the questionnaire. ${ }^{24-26}$ Then, the values of food items are converted to gram using the home volume guide, and nutrition software Nutritionist-4 has been used to analyze the intake of nutrients (micronutrients and macronutrients and energy intake).

\section{Calculation of DII}

DII was calculated based on Shivappa et al method using dietary intake data that provided by validated quantitative FFQ for each individual and the details of DII score calculation completely described elsewhere. ${ }^{11}$ According to this method after a comprehensive review of scientific literature for each of the 45 food parameters, the inflammatory score, mean and standard deviation (SD) of global intake were determined. To calculate DII, each of the 45 dietary parameters was first subtracted from the global mean of that dietary parameter and divided by its global standard deviation. This Z-score was standardized for its food parameter to minimize the right skew effect, then this value was converted to a percentile score and multiplied by two and subtracted from one. Subsequently, the obtained number multiplied by the corresponding inflammatory score for that food parameter. Finally, the sum of all calculated numbers from the 45 dietary parameters per person indicated the total inflammatory score for each individual. ${ }^{11}$ In this study out of 45 food parameters, only 31 parameters were used to calculate DII, including; vitamin $\mathrm{B} 12, \mathrm{~B} 6, \mathrm{E}, \mathrm{C}, \mathrm{A}, \mathrm{D}$, total fat, trans fat, monounsaturated fatty acids (MUFA), polyunsaturated fatty acids (PUFA), cholesterol, omega-3, omega-6, saturated fat, selenium, zinc, energy, iron, magnesium, niacin, riboflavin, thiamine, beta-carotene, carbohydrate, protein, fiber, folic acid, caffeine, garlic, onion and tea. Then, DII scores were classified into four groups (quartile) to investigate the relationship with the different variables. The first and fourth quartiles had the lowest and highest DII scores, respectively.

\section{Metabolic Syndrome}

MetS was detected based on NCEP-ATP III criteria. According to the NCEP-ATP III definition, MetS is present if three or more of the following five criteria are met; WC over 40 inches (men) or 35 inches (women), blood pressure over 130/85 mmHg, fasting TG level over $150 \mathrm{mg} / \mathrm{dl}$, fasting HDL-C level less than $40 \mathrm{mg} / \mathrm{dl}$ (men) or $50 \mathrm{mg} / \mathrm{dl}$ (women) and FBG over $110 \mathrm{mg} / \mathrm{dl}^{27}$

\section{Statistical Analyses}

For statistical analyses, the statistical software package (STATA-14) was used. Each subject was recorded with separate code for following analyses. Chi-square test was used to analyze the statistical significance of categorical variables (sex, MetS, marital status, education, economic status, smoking, physical activity and residential areas) in DII quartiles. Also, the statistical significance of continuous variables [age, BMI, total calorie intake, WC, TG, systolic blood pressure (SBP), diastolic blood pressure (DBP), HDL-C and FBG] across different quartiles of the DII 
were analyzed by One-way ANOVA method. The logistic regression model was performed to investigate the relationship between DII quartiles with MetS and its components. Besides the raw model (unadjusted), two other models (model 1 and model 2) were used for the analysis of the data. Model 1 was adjusted for age, sex, marital status, education, economic status, smoking, physical activity level and total calorie intake. Model 2 was adjusted for variables in model 1 plus BMI as confounding factors. Also, the first quartile with the lowest inflammatory index was considered as the reference. The significance level of $<0.05$ with $95 \%$ confidence intervals (CIs) was considered for statistical analysis.

\section{Results}

In this study, after considering the exclusion criteria, 6538 subjects including male $(55.2 \%)$ and female (44.8\%) participated in which $17.1 \%$ of them (all participants) had MetS. The remaining subjects $(3527,35 \%)$ were excluded from the study due to incomplete data, underlying disease and alcohol or drug abuse. The mean age and BMI of participants were $46.2 \pm 3.4$ and $23.9 \pm 2.8$, respectively. Also, the mean DII score of participants was $1.17 \pm 0.02$ and its corresponding minimum and maximum scores were -4.38 and 5.10, respectively (Table 1). According to Table 2 , the baseline characteristics of categorical variables in the quartiles of the DII were significantly different except for MetS between both sexes. Besides, the mean value of the DII was significantly higher in men in comparison to women $(1.64$ vs 0.79$)(\mathrm{p}<0.001)$ and married individuals with higher education, better economic status, smoker, more physical activity, and city dwellers had significantly higher DII scores in comparison with the first quartile $(\mathrm{p}<0.001)$. As well, a significant positive correlation was found between increased DII and MetS (Table 2).

The mean $\pm \mathrm{SD}$ values of continuous variables in each quartile are presented in Table 3. Individuals with higher DII scores were significantly younger and had higher BMI,

Table I Classification of Dietary Inflammatory Index

\begin{tabular}{|l|l|l|l|l|}
\hline DII & Participants (n) & Mean \pm SD & Min & Max \\
\hline Quartile I & 1635 & $-1.07 \pm 0.86$ & -4.38 & 0.07 \\
Quartile 2 & 1634 & $0.78 \pm 0.37$ & 0.07 & 1.37 \\
Quartile 3 & 1635 & $1.84 \pm 0.27$ & 1.37 & 2.35 \\
Quartile 4 & 1634 & $3.1 \pm 0.57$ & 2.35 & 5.10 \\
\hline
\end{tabular}

Note: Data presented as mean $\pm S D$.

Abbreviation: DIl, dietary inflammatory index.
TG, DBP, FBG and intake more calorie compared to lower quartiles. The percent of participants with high SBP, DBP, TG and FBG were $8.7 \%, 6 \%, 28.4 \%$, and $8.9 \%$, respectively. The percent of men and women with low HDL-C and high WC were $18.8 \%, 29.7 \%$ and $10.7 \%$, 62.6\%, respectively. Also, the difference between the first and fourth quartiles (between quartiles) for age, BMI, total calorie intake, WC, TG, SBP, DBP and HDL-C was significant.

Moreover, individuals with higher DII scores were at higher risk of MetS and this relationship remained significant after adjustment for confounding factors (OR trend: 1.08, 95\% CI: $1.01-1.15, \mathrm{P}=0.017)$. With increasing of DII score, a significant increase in TG (OR trend: 1.06, 95\% CI: 1.00-1.12, $\mathrm{P}=0.030$ ), FBG level (OR trend: 1.10, 95\% CI: $1.01-1.20, \mathrm{P}=0.018)$ and a significant decrease in HDL-C level (OR trend: 1.07, 95\% CI: 1.02-1.12, $\mathrm{P}=0.005$ ) were observed in model 2 . In addition, a significant relationship was found between DII score and WC (OR trend: 1.07, 95\% CI: $1.01-1.14, \mathrm{P}=0.016$ ) in model 1 (Table 4).

\section{Discussion}

MetS is a collection of conditions such as abdominal obesity, hypertension, elevated FBG and TG levels, decreased HDL-C level that may occur together and increase the risk of different chronic diseases like kidney disease, cardiovascular disease and diabetes. ${ }^{6,10,28,29}$ The genetic predisposition together with environmental factors such as dietary patterns, inactive lifestyles, high BMI and age are shown to be associated with increased prevalence of MetS. ${ }^{30}$ Since high inflammatory diets are recognized as one of the most important environmental factors in causing inflammation and predispose individuals to chronic inflammatory diseases such as MetS and diabetes, we investigated the association between DII and MetS and its components using data from 6538 people who participated in a cohort study in Iran. Our findings showed that individuals with higher DII scores were at higher risk for MetS and its components including elevated TG and FBG levels, decreased HDL-C and higher WC. Moreover, there was a significant association between DII and MetS in the general population, which is consistent with the results of Neufcourt et al that demonstrated higher DII scores were associated with higher SBP, DBP, TG, and lower HDL-C. ${ }^{2}$ Likewise, in a crosssectional study that consisted of 606 participants from EastAzerbaijan-Iran, higher DII score was significantly associated with MetS and after adjustment for smoking status, physical activity, sex, age and BMI, highest quartile of DII score had significantly higher FBG compared with the lowest quartile of DII score. ${ }^{31}$ In addition, our investigation showed that the 
Table 2 The Baseline Characteristics of Categorical Variables According to DII Quartiles

\begin{tabular}{|c|c|c|c|c|c|c|}
\hline \multirow[t]{2}{*}{ Variable (n) } & & \multicolumn{5}{|l|}{ DII Quartiles } \\
\hline & & QI $(n=1635)$ & Q2 $(n=1634)$ & Q3 $(n=1635)$ & Q4 $(n=1634)$ & $\mathbf{P} *$ \\
\hline \multirow[t]{2}{*}{ Sex } & Male & 443 & 653 & 880 & 955 & $<0.001$ \\
\hline & Female & 1192 & 981 & 755 & 679 & \\
\hline \multirow[t]{2}{*}{ MetS } & Male & 149 & 192 & 239 & 275 & 0.761 \\
\hline & Female & 329 & 303 & 228 & 186 & 0.053 \\
\hline \multirow[t]{3}{*}{ Marital status } & Single & 139 & 86 & 36 & 45 & $<0.001$ \\
\hline & Married & 1362 & 1463 & $|50|$ & 1530 & \\
\hline & Widow & 134 & 85 & 71 & 59 & \\
\hline \multirow[t]{5}{*}{ Education } & Illiterate & 619 & 392 & 318 & 264 & $<0.001$ \\
\hline & $\leq 5$ years & 684 & 703 & 634 & 589 & \\
\hline & 6-9 years & 192 & 249 & 306 & 337 & \\
\hline & $10-12$ years & 90 & 176 & 233 & 263 & \\
\hline & $\geq 13$ years & 50 & 113 & 144 & $|8|$ & \\
\hline \multirow[t]{5}{*}{ Economic status } & QI & 532 & 361 & 280 & 214 & $<0.001$ \\
\hline & Q2 & 398 & 367 & 318 & 281 & \\
\hline & Q3 & 317 & 325 & 355 & 304 & \\
\hline & Q4 & 237 & 283 & 361 & 403 & \\
\hline & Q5 & $|5|$ & 298 & 321 & 432 & \\
\hline \multirow[t]{2}{*}{ Smoking } & No & 1378 & 1347 & 1307 & 1293 & $<0.001$ \\
\hline & Yes & 257 & 287 & 328 & $34 I$ & \\
\hline \multirow[t]{3}{*}{ PAL } & Light & 396 & 406 & 432 & 397 & 0.001 \\
\hline & Moderate & 923 & 897 & 811 & 847 & \\
\hline & Intense & 316 & 331 & 392 & 390 & \\
\hline \multirow[t]{2}{*}{ Residential areas } & Urban & 579 & 857 & 1043 & 1278 & $<0.001$ \\
\hline & Rural & 1056 & 777 & 592 & 356 & \\
\hline
\end{tabular}

Note: *P-value was obtained by the Chi-square test and $\mathrm{P}<0.05$ were considered as statistically significant.

Abbreviations: Q, quartile; MetS, metabolic syndrome; DII, dietary inflammatory index; PAL, physical activity level.

Table 3 The Baseline Characteristics of Continuous Variables According to DII Quartiles

\begin{tabular}{|c|c|c|c|c|c|c|c|c|c|}
\hline \multirow[t]{3}{*}{ Variable (Mean \pm SD) } & \multicolumn{9}{|c|}{ DII Quartiles } \\
\hline & \multicolumn{2}{|c|}{ QI $(n=1635)$} & \multicolumn{2}{|c|}{ Q2 $(n=1634)$} & \multicolumn{2}{|c|}{ Q3 $(n=1635)$} & \multicolumn{2}{|c|}{ Q4 (n=1634) } & \multirow[t]{2}{*}{$\mathbf{P}^{\mathbf{a}}$} \\
\hline & Male & Female & Male & Female & Male & Female & Male & Female & \\
\hline Age (year) & $50.57 \pm 2.9$ & $48.52 \pm 2.3$ & $48.63 \pm 3.0$ & $47.08 \pm 4.8$ & $47.1 \pm 2.7$ & $46.2 \pm 6.1$ & $40.5 \pm 3.8$ & $41.6 \pm 2.3$ & 0.001 \\
\hline BMI $\left(\mathrm{kg} / \mathrm{m}^{2}\right)$ & $24.6 \pm 1.7$ & $25.3 \pm 2.2$ & $25.3 \pm 2.9$ & $28.3 \pm 3.2$ & $26 \pm 3.7$ & $28.6 \pm 1.4$ & $28.4 \pm 3.9$ & $29.5 \pm 3.9$ & 0.001 \\
\hline Total calorie intake $(\mathrm{Kcal})$ & $2321.7 \pm 55$ & $2072 \pm 56$ & $3162 \pm 62$ & $2836 \pm 55$ & $3829 \pm 89$ & $3382 \pm 62$ & $4526 \pm 94$ & $4066 \pm 103$ & 0.001 \\
\hline$W C(\mathrm{~cm})$ & $94.1 \pm 9.1$ & $96.3 \pm 10.4$ & $94.6 \pm 9.4$ & $97.7 \pm 11.3$ & $95.6 \pm 9.2$ & $98.2 \pm 10.2$ & $95.7 \pm 9.3$ & $98.7 \pm 9.5$ & 0.001 \\
\hline TG (mg/dl) & $138.9 \pm 12.3$ & $118.8 \pm 13.2$ & $132.2 \pm 16.3$ & $130.2 \pm 1 \mid .2$ & $138.8 \pm 1 \mid .7$ & $130.2 \pm 14.4$ & $148.4 \pm 16.2$ & $136.1 \pm 12.5$ & 0.03 \\
\hline $\mathrm{SBP}(\mathrm{mmHg})$ & $107.6 \pm 10.5$ & $106.7 \pm 8.5$ & $106.2 \pm 12.4$ & $107.3 \pm 9.5$ & $107.6 \pm 9.4$ & $107.1 \pm 10.9$ & $108.6 \pm 11$ & $108.3 \pm 11.7$ & 0.026 \\
\hline $\mathrm{DBP}(\mathrm{mmHg})$ & $69.8 \pm 9.8$ & $67.5 \pm 7.4$ & $68.8 \pm 8.8$ & $69.9 \pm 6.5$ & $69.7 \pm 9.4$ & $69.6 \pm 7.3$ & $79.8 \pm 9.9$ & $77.4 \pm 9.5$ & 0.021 \\
\hline HDL-C (mg/dl) & $44.2 \pm 4.2$ & $50.7 \pm 3.4$ & $43.5 \pm 5.8$ & $49.8 \pm 4.6$ & $43.4 \pm 2.3$ & $49 \pm 1.7$ & $42.5 \pm 3.6$ & $48.9 \pm 6.6$ & 0.001 \\
\hline FBG (mg/dl) & $93 \pm 8.8$ & $94.1 \pm 6.7$ & $94.6 \pm 9.4$ & $95.2 \pm 8$ & $90.8 \pm 10.2$ & $90.8 \pm 9.6$ & $105.5 \pm 9.2$ & $101.5 \pm 9.7$ & 0.996 \\
\hline
\end{tabular}

Note: ${ }^{a} \mathrm{P}$-value was obtained by the One-way ANOVA test between quartiles (the fourth quartile compared to the first quartile) and $\mathrm{P}<0.05$ were considered as statistically significant. Abbreviations: Q, quartile; BMI, body mass index; DII, dietary inflammatory index; WC, waist circumference; TG, triglyceride; SBP, systolic blood pressure; DPB, diastolic blood pressure; HDL-C, high-density lipoprotein cholesterol; FBG, fasting blood glucose.

male participants had a significantly higher DII score than females. Similarly, married subjects with higher education levels, better economic status, smoker, more physical activity, and city dwellers showed higher DII scores $(p<0.001)$. According to several studies on the western population, the DII score tends to be lower in married subjects who had higher 
Table 4 Findings from the Logistic Regression Models That Evaluated Metabolic Syndrome Components According to DII Quartiles

\begin{tabular}{|c|c|c|c|c|c|c|c|}
\hline \multirow[t]{3}{*}{ Variables } & \multirow[t]{3}{*}{ Models } & \multicolumn{6}{|l|}{ DII Quartiles } \\
\hline & & \multicolumn{6}{|c|}{ Odds Ratios, $95 \%$ Confidence Intervals } \\
\hline & & $Q 1^{a}(n=1635)$ & Q2 (n=1634) & Q3 $(n=1635)$ & Q4 (n=1634) & OR trend & P-trend \\
\hline \multirow[t]{3}{*}{ MetS } & Crude $^{\mathbf{b}}$ & I & $1.04(0.87-1.25)$ & $0.98(0.82-1.18)$ & $0.95(0.79-1.14)$ & $0.98(0.94-1.02)$ & 0.398 \\
\hline & Model I $I^{\mathbf{C}}$ & I & $1.31(1.08-1.61)$ & $1.53(1.21-1.92)$ & $1.66(1.26-2.18)$ & $1.14(1.07-1.22)$ & $<0.001$ \\
\hline & Model $2^{d}$ & I & $1.18(0.96-1.45)$ & $1.33(1.05-1.69)$ & $1.34(1.01-1.77)$ & $1.08(1.01-1.15)$ & 0.017 \\
\hline \multirow[t]{3}{*}{ High WC } & Crude & I & $0.83(0.72-0.96)$ & $0.63(0.54-0.72)$ & $0.54(0.47-0.62)$ & $0.86(0.83-0.89)$ & $<0.001$ \\
\hline & Model I & I & $1.20(0.99-1.45)$ & $1.30(1.04-1.61)$ & $1.17(0.91-1.52)$ & $1.07(1.01-1.14)$ & 0.016 \\
\hline & Model 2 & - & - & - & - & - & - \\
\hline \multirow[t]{3}{*}{ High TG } & Crude & I & $1.25(1.07-1.46)$ & $1.36(1.16-1.59)$ & $1.30(1.11-1.52)$ & $1.06(1.02-1.09)$ & $<0.001$ \\
\hline & Model I & I & $1.31(1.11-1.55)$ & $1.46(1.21-1.77)$ & $1.46(1.17-1.83)$ & $1.09(1.04-1.15)$ & $<0.001$ \\
\hline & Model 2 & 1 & $1.24(1.04-1.47)$ & $1.34(1.11-1.63)$ & $1.29(1.02-1.62)$ & $1.06(1.00-1.12)$ & 0.030 \\
\hline \multirow[t]{3}{*}{ High Hypertension } & Crude & 1 & $1.00(0.71-1.39)$ & $0.98(0.70-1.37)$ & $1.22(0.89-1.68)$ & $1.03(0.96-1.10)$ & 0.359 \\
\hline & Model I & 1 & $1.21(0.84-1.74)$ & $1.33(0.88-2.01)$ & $1.80(1.12-2.88)$ & $1.14(1.02-1.27)$ & 0.017 \\
\hline & Model 2 & 1 & $1.12(0.78-1.61)$ & $1.20(0.79-1.18)$ & $1.56(0.97-2.50)$ & $1.10(0.98-1.23)$ & 0.087 \\
\hline \multirow[t]{3}{*}{ Low HDL-C } & Crude & I & $1.10(0.96-1.27)$ & $1.09(0.95-1.25)$ & $1.14(1.00-1.31)$ & $1.03(1.00-1.06)$ & 0.021 \\
\hline & Model I & 1 & $1.19(1.02-1.38)$ & $1.27(1.07-1.5 \mathrm{I})$ & $1.39(1.14-1.71)$ & $1.10(1.05-1.16)$ & $<0.001$ \\
\hline & Model 2 & 1 & I.I3 (0.97-I.3I) & I.I7 (0.98-I.40) & $1.24(1.01-1.53)$ & $1.07(1.02-1.12)$ & 0.005 \\
\hline \multirow[t]{3}{*}{ High FBG } & Crude & 1 & $1.00(0.78-1.29)$ & $1.00(0.78-1.29)$ & $1.17(0.92-1.48)$ & $1.02(0.97-1.07)$ & 0.372 \\
\hline & Model I & I & $1.20(0.92-1.58)$ & $1.39(1.02-1.89)$ & $1.77(1.24-2.52)$ & $1.14(1.05-1.24)$ & 0.002 \\
\hline & Model 2 & I & $1.13(0.86-1.48)$ & $1.28(0.94-1.74)$ & $1.57(1.10-2.24)$ & $1.10(1.01-1.20)$ & 0.018 \\
\hline
\end{tabular}

Notes: The logistic regression test was used for analysis; ${ }^{a}$ As reference DII quartile; ${ }^{b}$ Unadjusted; ${ }^{c}$ Model I: Adjusted for age, sex, education, marital status, economic status,

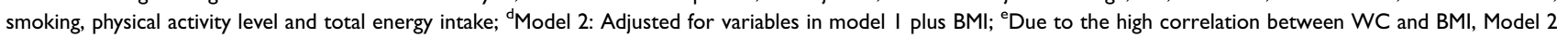
was not done for this component of MetS.

Abbreviations: Q, quartile; DII, dietary inflammatory index; MetS, metabolic syndrome; WC, waist circumference; TG, triglyceride; HDL-C, high-density lipoprotein cholesterol; FBG, fasting blood glucose.

economic status and higher education level which is inconsistent with our findings. ${ }^{2,16,32}$ It seems that in middle-income countries, city dweller families with increasing education level, following by higher income and social well-being, tend to consume more foods with high inflammatory index (fast foods and meats) and this may be due to their high occupational problems and also not having enough time to prepare healthy food. Also, in middle-income countries, the animal protein sources (red meat) are expensive, so people who live in city and have higher education level and income consume more animal sources than people living in rural areas rural. On the other hand, people living in rural areas due to adherence to their traditional foods, which are often healthy (vegetables and fruits) and have lower DII score, are less likely influence by MetS components and its related chronic disease.

Numerous studies showed that obesity and its consequences like MetS may cause an imbalance in adipokines profile. ${ }^{33,34}$ MetS as a chronic inflammatory situation may lead to increase of pro-inflammatory adipokines and cytokines level such as Leptin, IL-6, IL-12, IL-18, chemerin, retinol-binding protein 4 (RBP4), lipocalin 2 (LCN2), TNF- $\alpha$, and hs-CRP. By contrast, MetS can diminish the anti-inflammatory adipokines such as adiponectin, C1q/TNF-related protein (CTRP) family and Omentin-1. ${ }^{2}$ The formation of this pro-inflammatory condition that is positively correlated with abdominal obesity and high BMI can increase the reactive oxygen and nitrogen species levels and play a central role in the etiology of the MetS. ${ }^{35-37}$ In this way, DII score of the diet can be a strong stimulator or modulator of chronic and systemic inflammation. ${ }^{28}$ Unhealthy diet and its components are the most important factors that prompt inflammatory pathways. Various studies have been demonstrated that following a healthy diet, which is contained from fruits and vegetables, is associated with lower plasma concentrations of CRP, homocysteine, ICAM1, IL1R1, IL6, E-selectin, sVCAM-1 TNF- $\alpha$ and NF- $\kappa B 1$ as inflammatory factors. ${ }^{12,38}$ A growing body of evidence demonstrated that the DII score in some healthy dietary patterns such as DASH (dietary approach to stop hypertension), AHEI (alternate healthy eating index) and HEI-2010 
(healthy eating index-2010) is lower than other unhealthy patterns. ${ }^{32,39}$

The beneficial role of Mediterranean diet which is based on fruits, vegetables, olive oil, fish, nuts, legumes, whole grains, low-fat dairy products and low alcohol intake has been proved in metabolic disease and CVD. On the other hand, lower intake of red meat and saturated fat as the profitable properties of this diet showed favorable effects on the blood pressure level and BMI. ${ }^{31,40}$ The Mediterranean diet can also modulate the systemic inflammation and coagulation process and attenuate the progress and development of inflammatory diseases and cancers. ${ }^{41-43}$ The best explanation for these beneficial effects is the existence of some important nutrients like n-3 PUFA, ${ }^{12,44}$ fiber, ${ }^{45-47}$ vitamin $\mathrm{E},{ }^{48-50}$ vita$\min C,^{51,52} \beta$-carotene ${ }^{51,53,54}$ and magnesium ${ }^{45,55}$ that mostly possess lower DII scores and prevent from inflammation and perform as antioxidants which inhibit the production of free radicals. ${ }^{15}$

On the other hand, different studies showed that individuals who consume western diet with high-refined grains, simple carbohydrates, butter, red meat, processed meat, sweets, and soft drinks have higher BMI, sedentary lifestyle, energy intake, serum cholesterol, CRP and IL-6 levels and also have lower intake of vitamin B-6, magnesium, and fiber. ${ }^{12}$ Esmaillzadeh et al revealed that the western dietary pattern score is significantly associated with inflammatory markers and after adjustment for age, BMI, smoking, physical activity, current estrogen use, menopausal status, family history of diabetes, stroke and energy intake this relationship remained significant for CRP, IL-6, and serum amyloid A (SAA). ${ }^{12}$ In the same way, our results showed that MetS and its components were significantly associated with DII score and this relationship remained significant after adjustment for main confounding factors like age, sex, education, marital status, economic status, smoking, physical activity level and total energy intake (Table 4).

Various investigations confirmed the potential role of the diet on the development and progress of cardiovascular disease and atherosclerosis through inducing inflammatory markers like CRP, IL-6, sICAM-1, sVCAM-1, and E-selectin. The findings of Nurses' Health Studies and Health Professionals Follow-up Study suggested that prudent dietary pattern with higher fruits, vegetables, legumes, fish, poultry, and whole grains have a reverse association with markers of inflammation (CRP), endothelial dysfunction (E-selectin), insulin, and homocysteine concentrations. ${ }^{56-58}$ Moreover, Nettleton et al in the Multi-Ethnic Study of
Atherosclerosis (MESA), using factor analysis on an ethnically diverse population, recognized four dietary patterns that were comparable with prudent and western diet patterns in other populations. The identified dietary patterns were positively associated with serum CRP, homocysteine and sICAM-1 levels. ${ }^{59}$ Similarly, in the present study, we showed that the components of the western dietary pattern with higher DII scores that are consumed by the Iranian population can be an effective risk factor for atherosclerosis through increasing TG and FBG and decreasing HDL-C level. Also, our findings revealed that participants with higher DII scores were younger and significantly had higher BMI, total calorie intake and DBP levels. These findings were consistent with the results of two different cross-sectional and case-control studies performed on Iranian populations. ${ }^{31,60}$

The primary strength of this study was the large sample size for both sexes that evaluated the data from the cohort study. Using DII score as a validated instrument to evaluate the inflammatory impacts of diet, evaluation of diet by validated $\mathrm{FFQ}$, using physical activity questionnaire and also adjustment for a large number of confounding factors that associated with MetS were the other pros of the current study. However, there were some limitations regarding the methodology and nature of the present study. The primary limitation was the number of dietary parameters so that only 31 dietary parameters were used to calculate DII score. Moreover, due to the cross-sectional nature of the study, we were unable to infer causality. Also, using FFQ as a retrospective dietary assessment method, which is based on the subjects' memory, increases the possible misreporting and can be considered as other limitation of our study.

\section{Conclusion}

In summary, the present study was the first large population study in Iran that assessed the association between dietary inflammatory parameters with MetS. The findings of our study showed a significant association between DII and MetS, WC, TG, hypertension, FBG and a reverse association with HDL-C after adjustment for different covariates. Knowing the association of DII with MetS components and some anthropometric indices, the DII can be suggested as a suitable clinical instrument to determine pro-inflammatory components of the diet to ultimately reduce the risk of chronic non-communicable disease. However, further longitudinal investigations with more dietary parameters are needed to fully understand the 
role of inflammatory diets with an increased risk of MetS and its components.

\section{Abbreviations}

AHEI, alternate healthy eating index; BMI, body mass index; CAM, cell adhesion molecules; CVD, cardiovascular disease; DASH, dietary approach to stop hypertension; DII, dietary inflammatory index; FFQ, food frequency questionnaire; HDL-C, high-density lipoprotein cholesterol; HEI-2010, healthy eating index-2010; hs-CRP, high-sensitivity C-reactive protein; IL, interleukin; IPAQ, international physical activity questionnaire; LDL-C, low-density lipoproteins cholesterol; MetS, metabolic syndrome; NCEP/ATPIII, national cholesterol education program/adult treatment panel III; RaNCD, Ravansar non-communicable diseases; MUFA, monounsaturated fatty acids; MESA, multi-ethnic study of atherosclerosis; PUFA, polyunsaturated fatty acids; SAA, serum amyloid A; TG, triglyceride; TNF- $\alpha$, tumor necrosis factor- $\alpha$; WC, waist circumference.

\section{Ethics Approval and Data Sharing Statement}

Ethical approval for the study was obtained from the Kermanshah University of Medical Sciences Ethics Committee. All authors confirmed that the data accessed was deidentified.

\section{Acknowledgment}

We are grateful to all the participants who took part in this study. This research did not receive any specific grant from funding agencies in the public, commercial, or not-for-profit sectors. The financial support of the Kermanshah University of Medical Sciences is gratefully acknowledged.

\section{Author Contributions}

All authors made substantial contributions to conception and design, acquisition of data, or analysis and interpretation of data; took part in drafting the article or revising it critically for important intellectual content; gave final approval of the version to be published; and agree to be accountable for all aspects of the work.

\section{Disclosure}

The authors report no conflicts of interest in this work.

\section{References}

1. Kassi E, Pervanidou P, Kaltsas G, et al. Metabolic syndrome: definitions and controversies. BMC Med. 2011;9(1):48. doi:10.1186/17417015-9-48

2. Neufcourt L, Assmann KE, Fezeu LK, et al. Prospective association between the dietary inflammatory index and metabolic syndrome: findings from the SU.VI.MAX study. Nutr Metab Cardiovasc Dis. 2015;25(11):988-996. doi:10.1016/j.numecd.2015.09.002

3. Kaur J. A comprehensive review on metabolic syndrome. Cardiol Res Pract. 2014;2014:943162. doi:10.1155/2014/943162

4. McCracken E, Monaghan M, Sreenivasan S. Pathophysiology of the metabolic syndrome. Clin Dermatol. 2018;36(1):14-20. doi:10.1016/ j.clindermatol.2017.09.004

5. Dalvand S, Niksima SH, Meshkani R, et al. Prevalence of metabolic syndrome among Iranian population: a systematic review and meta-analysis. Iran J Public Health. 2017;46(4):456-467.

6. Noshad S, Abbasi M, Etemad K, et al. Prevalence of metabolic syndrome in Iran: a 2011 update. J Diabetes. 2017;9(5):518-525. doi:10.1111/1753-0407.12438

7. Richard C, Couture P, Desroches S, et al. Effect of the Mediterranean diet with and without weight loss on markers of inflammation in men with metabolic syndrome. Obesity (Silver Spring). 2013;21(1):51-57. doi:10.1002/oby.20239

8. Shivappa N, Prizment AE, Blair CK, et al. Dietary inflammatory index and risk of colorectal cancer in the Iowa Women's Health Study. Cancer Epidemiol Biomarkers Prev. 2014;23(11):2383-2392. doi:10.1158/1055-9965.epi-14-0537

9. Bullo M, Casas-Agustench P, Amigo-Correig $P$, et al. Inflammation, obesity and comorbidities: the role of diet. Public Health Nutr. 2007;10(10a):1164-1172. doi:10.1017/s1368980007 000663

10. Ahluwalia N, Andreeva VA, Kesse-Guyot E, et al. Dietary patterns, inflammation and the metabolic syndrome. Diabetes \& Metabolism. 2013;39(2):99-110. doi:10.1016/j.diabet.2012.08.007

11. Shivappa N, Steck SE, Hurley TG, et al. Designing and developing a literature-derived, population-based dietary inflammatory index. Public Health Nutr. 2014;17(8):1689-1696. doi:10.1017/s1368980013002115

12. Esmaillzadeh A, Kimiagar M, Mehrabi Y, et al. Dietary patterns and markers of systemic inflammation among Iranian women. $J$ Nutr. 2007;137(4):992-998. doi:10.1093/jn/137.4.992

13. Barbaresko J, Koch M, Schulze MB, et al. Dietary pattern analysis and biomarkers of low-grade inflammation: a systematic literature review. Nutr Rev. 2013;71(8):511-527. doi:10.1111/nure.12035

14. Neale EP, Batterham MJ, Tapsell LC. Consumption of a healthy dietary pattern results in significant reductions in C-reactive protein levels in adults: a meta-analysis. Nutr Res. 2016;36(5):391-401. doi:10.1016/j.nutres.2016.02.009

15. Shivappa N, Steck SE, Hurley TG, et al. A population-based dietary inflammatory index predicts levels of C-reactive protein in the Seasonal Variation of Blood Cholesterol Study (SEASONS). Public Health Nutr. 2014;17(8):1825-1833. doi:10.1017/s1368980013002565

16. Wirth MD, Burch J, Shivappa N, et al. Association of a dietary inflammatory index with inflammatory indices and metabolic syndrome among police officers. J Occup Environ Med. 2014;56 (9):986-989. doi:10.1097/jom.0000000000000213

17. Medina-Remon A, Casas R, Tressserra-Rimbau A, et al. Polyphenol intake from a Mediterranean diet decreases inflammatory biomarkers related to atherosclerosis: a substudy of the PREDIMED trial. $\mathrm{Br}$ J Clin Pharmacol. 2017;83(1):114-128. doi:10.1111/bcp.12986

18. Urpi-Sarda M, Casas R, Chiva-Blanch G, et al. The Mediterranean diet pattern and its main components are associated with lower plasma concentrations of tumor necrosis factor receptor 60 in patients at high risk for cardiovascular disease. J Nutr. 2012;142(6):1019-1025. doi:10.3945/jn.111.148726 
19. Ley SH, Sun Q, Willett WC, et al. Associations between red meat intake and biomarkers of inflammation and glucose metabolism in women. Am J Clin Nutr. 2014;99(2):352-360. doi:10.3945/ ajcn.113.075663

20. Herieka M, Faraj TA, Erridge C. Reduced dietary intake of pro-inflammatory Toll-like receptor stimulants favourably modifies markers of cardiometabolic risk in healthy men. Nutr Metab Cardiovasc Dis. 2016;26(3):194-200. doi:10.1016/j.numecd.2015.12.001

21. Pasdar Y, Najafi F, Moradinazar M, et al. Cohort profile: Ravansar non-communicable disease cohort study: the first cohort study in a Kurdish population. Int $J$ Epidemiol. 2019;48(3):682f-683f. doi:10.1093/ije/dyy296

22. Poustchi H, Eghtesad S, Kamangar F, et al. Prospective epidemiological research studies in Iran (the PERSIAN cohort study): rationale, objectives, and design. Am J Epidemiol. 2018;187(4):647-655. doi:10.1093/aje/kwx314

23. Panel on Detection, Evaluation, and Treatment of High Blood Cholesterol in Adults E. Executive Summary of The Third Report of The National Cholesterol Education Program (NCEP) expert panel on detection, evaluation, and treatment of high blood cholesterol in adults (adult treatment panel III). JAMA. 2001;285(19):2486-2497. doi:10.1001/jama.285.19.2486

24. Moradi S, Pasdar Y, Hamzeh B, et al. Comparison of 3 nutritional questionnaires to determine energy intake accuracy in Iranian adults. Clin Nutr Res. 2018;7(3):213-222. doi:10.7762/cnr.2018.7.3.213

25. Malekshah A, Kimiagar M, Saadatian-Elahi M, et al. Validity and reliability of a new food frequency questionnaire compared to $24 \mathrm{~h}$ recalls and biochemical measurements: pilot phase of Golestan cohort study of esophageal cancer. Eur J Clin Nutr. 2006;60:971-977. doi:10.1038/sj.ejen.1602407

26. Mirmiran P, Esfahani FH, Mehrabi Y, et al. Reliability and relative validity of an FFQ for nutrients in the Tehran lipid and glucose study. Public Health Nutr. 2010;13(5):654-662. doi:10.1017/s1368980009991698

27. Parikh RM, Mohan V. Changing definitions of metabolic syndrome. Indian J Endocrinol Metab. 2012;16(1):7-12. doi:10.4103/22308210.91175

28. Sokol A, Wirth MD, Manczuk M, et al. Association between the dietary inflammatory index, waist-to-hip ratio and metabolic syndrome. Nutr Res. 2016;36(11):1298-1303. doi:10.1016/j.nutres.2016.04.004

29. Wannamethee SG, Shaper AG, Lennon L, et al. Metabolic syndrome vs Framingham risk score for prediction of coronary heart disease, stroke, and type 2 diabetes mellitus. Arch Intern Med. 2005;165 (22):2644-2650. doi:10.1001/archinte.165.22.2644

30. Kolovou GD, Anagnostopoulou KK, Salpea KD, et al. The prevalence of metabolic syndrome in various populations. Am J Med Sci. 2007;333(6):362-371. doi:10.1097/MAJ.0b013e318065c3a1

31. Nikniaz L, Nikniaz Z, Shivappa N, et al. The association between dietary inflammatory index and metabolic syndrome components in Iranian adults. Prim Care Diabetes. 2018;12(5):467-472. doi:10.1016/j.pcd.2018.07.008

32. Ruiz-Canela M, Zazpe I, Shivappa N, et al. Dietary inflammatory index and anthropometric measures of obesity in a population sample at high cardiovascular risk from the PREDIMED (PREvencion con DIeta MEDiterranea) trial. $B r \quad J \quad$ Nutr. 2015;113(6):984-995. doi:10.1017/s0007114514004401

33. Deng Y, Scherer PE. Adipokines as novel biomarkers and regulators of the metabolic syndrome. Ann N Y Acad Sci. 2010;1212:E1-e19. doi:10.1111/j.1749-6632.2010.05875.x

34. Kumari R, Kumar S, Kant R. An update on metabolic syndrome: metabolic risk markers and adipokines in the development of metabolic syndrome. Diabetes Metab Syndr. 2019;13(4):2409-2417. doi:10.1016/j.dsx.2019.06.005

35. Andersen CJ, Fernandez ML. Dietary strategies to reduce metabolic syndrome. Rev Endocr Metab Disord. 2013;14(3):241-254. doi:10.10 07/s11154-013-9251-y
36. Calder PC, Ahluwalia N, Brouns F, et al. Dietary factors and low-grade inflammation in relation to overweight and obesity. $\mathrm{Br}$ J Nutr. 2011;106(S3):S1-S78. doi:10.1017/S0007114511005460

37. Tataranni PA. Metabolic syndrome: is there a pathophysiological common denominator? World Rev Nutr Diet. 2005;94:75-83. doi:10.1159/000088221

38. Ritchie SA, Connell JM. The link between abdominal obesity, metabolic syndrome and cardiovascular disease. Nutr Metab Cardiovasc Dis. 2007;17(4):319-326. doi:10.1016/j.numecd.2006.07.005

39. Wirth MD, Hébert JR, Shivappa N, et al. Anti-inflammatory dietary inflammatory index scores are associated with healthier scores on other dietary indices. Nutr Res. 2016;36(3):214-219. doi:10.1016/j. nutres.2015.11.009

40. Pickup JC. Inflammation and activated innate immunity in the pathogenesis of type 2 diabetes. Diabetes Care. 2004;27(3):813-823. doi:10.2337/diacare.27.3.813

41. Kafatos A, Diacatou A, Voukiklaris G, et al. Heart disease risk-factor status and dietary changes in the Cretan population over the past 30 y: the seven countries study. Am J Clin Nutr. 1997;65(6):1882-1886. doi:10.1093/ajcn/65.6.1882

42. Panagiotakos DB, Pitsavos CH, Chrysohoou C, et al. Status and management of hypertension in Greece: role of the adoption of a Mediterranean diet: the Attica study. J Hypertens. 2003;21 (8):1483-1489. doi:10.1097/00004872-200308000-00011

43. Chrysohoou C, Panagiotakos DB, Pitsavos C, et al. Adherence to the Mediterranean diet attenuates inflammation and coagulation process in healthy adults: the ATTICA study. J Am Coll Cardiol. 2004;44 (1):152-158. doi:10.1016/j.jacc.2004.03.039

44. Pimenta AM, Toledo E, Rodriguez-Diez MC, et al. Dietary indexes, food patterns and incidence of metabolic syndrome in a Mediterranean cohort: the SUN project. Clin Nutr. 2015;34(3):508-514. doi:10.1016/ j.clnu.2014.06.002

45. Bo S, Durazzo M, Guidi S, et al. Dietary magnesium and fiber intakes and inflammatory and metabolic indicators in middle-aged subjects from a population-based cohort. Am J Clin Nutr. 2006;84 (5):1062-1069. doi:10.1093/ajcn/84.5.1062

46. Ajani UA, Ford ES, Mokdad AH. Dietary fiber and C-reactive protein: findings from national health and nutrition examination survey data. J Nutr. 2004;134(5):1181-1185. doi:10.1093/jn/134.5.1181

47. King DE, Egan BM, Woolson RF, et al. Effect of a high-fiber diet vs a fiber-supplemented diet on C-reactive protein level. Arch Intern Med. 2007;167(5):502-506. doi:10.1001/archinte.167.5.502

48. Murphy RT, Foley JB, Tome MT, et al. Vitamin E modulation of $\mathrm{C}$-reactive protein in smokers with acute coronary syndromes. Free Radic Biol Med. 2004;36(8):959-965. doi:10.1016/j.freeradbiomed. 2004.01.018

49. Imhof A, Froehlich M, Brenner H, et al. Effect of alcohol consumption on systemic markers of inflammation. Lancet. 2001;357 (9258):763-767. doi:10.1016/s0140-6736(00)04170-2

50. Upritchard JE, Sutherland WH, Mann JI. Effect of supplementation with tomato juice, vitamin $\mathrm{E}$, and vitamin $\mathrm{C}$ on LDL oxidation and products of inflammatory activity in type 2 diabetes. Diabetes Care. 2000;23(6):733-738. doi:10.2337/diacare.23.6.733

51. van Herpen-broekmans WM, Klopping-Ketelaars IA, Bots ML, et al. Serum carotenoids and vitamins in relation to markers of endothelial function and inflammation. Eur J Epidemiol. 2004;19(10):915-921. doi:10.1007/s10654-004-5760-z

52. Wannamethee SG, Lowe GD, Rumley A, et al. Associations of vitamin $\mathrm{C}$ status, fruit and vegetable intakes, and markers of inflammation and hemostasis. Am J Clin Nutr. 2006;83(3):567-574; quiz 726-567. doi:10.1093/ajcn.83.3.567

53. Gunter MJ, Stolzenberg-Solomon R, Cross AJ, et al. A prospective study of serum C-reactive protein and colorectal cancer risk in men. Cancer Res. 2006;66(4):2483-2487. doi:10.1158/0008-5472.can-053631 
54. Erlinger TP, Guallar E, Miller ER 3rd, et al. Relationship between systemic markers of inflammation and serum beta-carotene levels. Arch Intern Med. 2001;161(15):1903-1908. doi:10.1001/archinte.161.15.1903

55. Song Y, Li TY, van Dam RM, et al. Magnesium intake and plasma concentrations of markers of systemic inflammation and endothelial dysfunction in women. Am J Clin Nutr. 2007;85(4):1068-1074. doi:10.1093/ajen/85.4.1068

56. Lopez-Garcia E, Schulze MB, Fung TT, et al. Major dietary patterns are related to plasma concentrations of markers of inflammation and endothelial dysfunction. Am J Clin Nutr. 2004;80(4):1029-1035. doi:10.1093/ajen/80.4.1029

57. Schulze MB, Hoffmann K, Manson JE, et al. Dietary pattern, inflammation, and incidence of type 2 diabetes in women. Am J Clin Nutr. 2005;82(3):675-684; quiz 714-675. doi:10.1093/ajen.82.3.675
58. Fung TT, Rimm EB, Spiegelman D, et al. Association between dietary patterns and plasma biomarkers of obesity and cardiovascular disease risk. Am J Clin Nutr. 2001;73(1):61-67. doi:10.1093/ajcn/ 73.1 .61

59. Nettleton JA, Polak JF, Tracy R, et al. Dietary patterns and incident cardiovascular disease in the multi-ethnic study of atherosclerosis. Am J Clin Nutr. 2009;90(3):647-654. doi:10.3945/ajcn.2009.27597

60. Vahid F, Shivappa N, Karamati M, et al. Association between Dietary Inflammatory Index (DII) and risk of prediabetes: a case-control study. Appl Physiol Nutr Metab. 2017;42(4):399-404. doi:10.1139/ apnm-2016-0395

\section{Publish your work in this journal}

Diabetes, Metabolic Syndrome and Obesity: Targets and Therapy is an international, peer-reviewed open-access journal committed to the rapid publication of the latest laboratory and clinical findings in the fields of diabetes, metabolic syndrome and obesity research. Original research, review, case reports, hypothesis formation, expert opinion and commentaries are all considered for publication. The manuscript management system is completely online and includes a very quick and fair peer-review system, which is all easy to use. Visit http://www.dovepress.com/testimonials.php to read real quotes from published authors. 